\title{
Cardiac rehabilitation protocols in the elderly
}

\author{
Maria Vittoria Silverii, Alessandra Pratesi, Giulia Lucarelli, Francesco Fattirolli \\ Department of Experimental and Clinical Medicine, University of Florence, Azienda Ospedaliero Universitaria \\ Careggi, Florence, Italy
}

\begin{abstract}
Cardiac rehabilitation (CR) is a comprehensive multidisciplinary program individually tailored to the needs of patients with cardiovascular disease. Cardiovascular disease is prevalent in older adults and is the leading cause of death and major disability in adults $\geq 75$ years of age. The mean age of patients eligible for CR is increasing, with greater complexity and specific geriatric features, such as multimorbidity, frailty, and disability. In this population, CR interventions should be aimed to prevent disability and preserve the residual functional capacity. Every patient should be assessed with a multidimensional evaluation that includes clinical, functional, emotional, cognitive and social domains.

Exercise-based CR programs have shown to be effective in
\end{abstract}

Correspondence: Prof. Francesco Fattirolli, Department of

Experimental and Clinical Medicine, University of Florence and Azienda Ospedaliero Universitaria Careggi, Cardiac Rehabilitation

Unit, Florence, Italy.

Tel. +39.055.7949558.

E-mail: francesco.fattirolli@unifi.it

Key words: Older adults; cardiac rehabilitation; frailty.

Contributions: All the authors made a substantive intellectual contribution All the authors have read and approved the final version of the manuscript and agreed to be accountable for all aspects of the work.

Conflict of interest: The authors declare that they have no competing interests, and all authors confirm accuracy.

Ethics approval and consent to participate: Not applicable.

Patient consent for publication: Not applicable.

Informed consent: The manuscript does not contain any individual person's data in any form.

Received for publication: 19 February 2020.

Accepted for publication: 7 May 2020.

${ }^{\circ}$ Copyright: the Author(s), 2020

Licensee PAGEPress, Italy

Monaldi Archives for Chest Disease 2020; 90:1253

doi: $10.4081 /$ monaldi.2020.1253

This article is distributed under the terms of the Creative Commons Attribution Noncommercial License (by-nc 4.0) which permits any noncommercial use, distribution, and reproduction in any medium, provided the original author(s) and source are credited. improving function and quality of life, by reducing disability and age-related deconditioning and contributing favorably to improved health outcomes in an aged population. Very old and frail patients seem to get an even greater potential benefit, and an early start after an acute event can prevent the post-hospital syndrome. Despite these proven benefits, CR is often underused in this population and a great effort should be done to encourage them to attend these programs.

There are just a few studies about CR programs in very old and frail patients, therefore a future goal should be to fill this gap.

\section{Introduction}

Cardiac rehabilitation (CR) is a comprehensive long-term program involving exercise training as well as medical evaluation, cardiac risk factors modification, education, and counseling. CR is designed to limit the physiological and psychological effects of cardiac illness, reduce the risk of sudden death or reinfarction, control cardiac symptoms, stabilize or reverse the atherosclerotic process, and enhance the psychosocial and vocational status of patients. CR programs also provide opportunities to review medications and therapeutic goals and to ensure that the management is well-directed, well-coordinated, and patientcentered. Several reviews have recently been published reinforcing the efficacy of CR but the number of very old patients ( $>75$ years old) included is limited, with few and small randomized clinical trials and observational studies available [1].

\section{Cardiovascular disease in the elderly}

Cardiovascular disease (CVD) is prevalent in older adults: thanks to improvements in surgical and interventional cardiology, more elderly are surviving after acute events. The prevalence of coronary diseases, heart failure, and peripheral arterial disease increases with age, as does the prevalence of diabetes mellitus, chronic obstructive pulmonary disease, chronic kidney disease, arthritis, anemia, mild cognitive impairment, dementia, and other diseases that often occur concurrently with CVD and compound management complexity. Many of the detrimental effects of aging and associated diseases are linked to sedentary behaviors, which in turn escalate the progression of aggregate vulnerability. Acute disease and hospitalization can accelerate these risks, with progressive disability associated with each cardiac exacerbation, leading to even more downstream risks of recurrent disease, institutionalization, and mortality. Therefore, there is a growing population of elderly cardiac patients, who are complex and heterogeneous with their specific geriatric features such as 
multimorbidity, frailty, and disability. CVD and hospitalization can further exacerbate age-related conditions and loss of function. While CR programs were originally designed to carry out secondary prevention in middle-aged patients, are current $\mathrm{CR}$ programs suitable for managing the old and very old patient?

\section{Frailty and cardiac elderly patients}

Frailty is a geriatric syndrome characterized by a variable combination of muscle weakness, mobility impairment, physical inactivity, social isolation, cognitive impairment, mood disturbance, and fatigue. Frailty is related to medical outcomes in the elderly, independently of how measured, and is predictive of hospitalization, institutionalization, falls, and worsening health status [2]. The association between frailty and mortality has been confirmed in many studies and across various settings and subpopulations $[3,4]$. It is a reversible condition if treated early and with appropriate interventions [5]. Two main models of frailty have been proposed. According to the phenotype model proposed by Fried et al., frailty is defined as a biological syndrome resulting from cumulative decline across multiple physiological systems [6]. Rockwood et al. proposed a model of frailty as a multidimensional risk state measured based on more by the deficits' accumulation [7]. Despite the relevance of frailty as a prognostic indicator, uncertainty remains regarding its definition, its measurement, the feasibility of its measurement in clinical practice, and whether such measurements can be influenced by interventions or describe the progress or deterioration of health status [8]. The biologically interconnected symptoms and signs that are diagnostic elements of the frailty phenotype (i.e., weakness, slow gait speed, low physical activity, exhaustion, unintentional weight loss) jointly express energy dysregulation. The escalating prevalence of frailty in the old age is associated with the mounting physiological dysregulation with aging; the association is non-linear and when frailty is present, stress responses are highly dysregulated and there is high vulnerability to adverse outcomes. In a recent position paper from the European Association for Preventive Cardiology, the Authors suggested the importance of frailty measurement in CR to help plan patients' management and estimate their prognosis, even if the prevalence of frailty and its clinical and prognostic relevance have not been well characterized in this environment yet [8]. Several reviews came to the same conclusion that exercise has beneficial effects in frail older adults: the exercise may be the medicine that can prevent frailty as well as reverse it, or at the very least it can slow the accumulation of functional deficits [9]. Research has not yet conclusively identified the optimal exercise program for reversing frailty concerning frequency, type, and duration of the exercise.

\section{Cardiac rehabilitation in the elderly}

According to a recent Position Paper of the Italian Association for Cardiovascular Prevention and Rehabilitation, CR intervention in the elderly must be very differentiated, taking into account the specific needs of the individual and focusing on the most significant interventions for each subject. Every patient should be assessed by a multidimensional evaluation that includes clinical, functional, emotional, cognitive, and social domains [11]. Interventions should be effective and sustainable but also involve patients and respect their preferences and expectations. To achieve the inclusion of elderly patients, CR programs should face the relative barriers to their access, including the lack of patient motivation, facilities' accessibility, social/family support [11].

CR provides a unique opportunity to review medications, reducing polypharmacy (commonly defined as the use of at least 5 medications), checking drug interactions, improving adherence and self-administration [12]. Nutritional status and eating behavior have a relevant role in the secondary prevention of CVD in patients undergoing CR: in the elderly, the nutritional assessment should focus on risk factors for malnutrition and sarcopenia, such as edentulism and hyporexia, but also solitude, isolation, economic conditions, social deprivation. The assessment of the nutritional status requires several diagnostic methods and indicators: clinics, biohumoral, anthropometric, and instrumental evaluation. The Mini Nutritional Assessment (MNA) is a screening tool and it is useful to identify multifactorial causes of nutritional risk specifically in the elderly. It consists of a questionnaire of 18 components grouped into four main ones, which are anthropometry data, general status, dietary habits, self-perceived health, and nutrition states [13-15]. Early initiation of CR is particularly important in these patients to break the cycle of the conditioning and post-hospital syndrome [9]. The greater the level of physical impairment, the greater the potential benefit of CR $[11,16]$; these data are particularly relevant since clinicians and researchers frequently dismiss frail patients as "too unfit for", concerned about their inability to complete a standard exercise program [10]. In Italy, the mean age of patients eligible for CR is increasing: at present, patients older than 75 years represent about one-third of those referred to CR [17]. Psychological support represents a crucial intervention in CR since cardiovascular diseases are closely related to psychological factors such as depression and anxiety. Specifically, in older adults, depressive disorders and anxiety can lead to limited physical function and decreased ability to perform daily life activities, worsening the quality of life and the adherence to secondary prevention measures [18-20]. Some randomized trials have shown that psychological interventions can improve the mood in adults with cardiac disease, but evidence of efficacy in older age are still few. Future large-scale trials testing the effectiveness of psychological therapies are required in consequence of the evidence uncertainty [21].

Even among older patients, CR has proven to be effective in reducing mortality, improving exercise capacity, and quality of life [22]. Suaya et al. studied over 600.000 Medicare patients after coronary heart disease and showed that CR benefits significantly reduce mortality at 1 year -with a progressively greater absolute difference in mortality at 5 years- and that the benefits persisted even in the very old with comorbidities [23]. Cochrane reviews demonstrated that exercise training may reduce mortality and improve self-reported quality of life in patients with heart failure, including the older and frail ones [24,25]. In a recent study, CR demonstrated to be effective in improving quality of life, functional capacity, and reducing frailty, after transcatheter aortic valve implantation in very old patients [26].

\section{Functional status}

Functional decline, defined as a new reduction in the ability to perform self-care activities of daily living (ADL), occurs frequently in older persons with CVD, and it is related to greater mortality $[27,28]$. Patients with a moderate or severe disability had a 1.8- and 4.5- fold increased risk of overall and fatal coronary heart disease 
respectively compared to nondisabled subjects [29]. On the other hand, in previously independent persons, a myocardial infarction (MI) increases functional impairment with greater longitudinal changes than those before MI and with a linear increase of 0.14 limitations/year in the following decade [30].

Aging encompasses systemic, organ-level, and cellular changes that predispose individuals to cardiovascular and comorbid diseases and geriatric syndromes with a progression that can be insidious, debilitating, and harmful. Functional decline is integral to these patterns. Adults who survive into old age are predictably more vulnerable to become sedentary and to start an exercise program wherein multimorbidities and hospitalizations compound functional decrements, which exacerbate risks of disability, dependency, and frailty. Whereas most major randomized controlled trials of therapies for CVD are oriented to outcomes metrics of mortality, morbidity, and hospitalizations, they rarely address the associated risks of functional loss that can occur even if the initial therapies seem to work out. Many older patients regard preserved function, independence, and related self-efficacy as their primary goals of care; that is, for many patients conserved functional capabilities become more important than traditional disease-specific therapeutic endpoints [31].

CR represents the ideal setting to assess the functional status in elderly cardiac patients $[11,32]$ and may play a crucial role in preventing decline and in lengthening disability-free survival following a cardiac event. Using information about cognition, mobility, function, and co-morbidities based on the history and physical examination, the health professional should be able to assess the patient frailty level (Table 1) [7, 33].

\section{Physical performance evaluation}

Evaluation of physical capacity is needed in CR programs to define the goals of intervention at the functional level.

Peak oxygen uptake (peak $\mathrm{VO}_{2}$ ) measured by cardiopulmonary exercise test (CPET) is the gold standard to evaluate exercise capacity [31], and every patient should be evaluated by CPET at CR program admission. CPET allows not only to assess the real extent of the reduction in functional capacity but also to identify alterations of the cardiovascular, respiratory or skeletal muscle system, as well as to evaluate the hemodynamic effects of drug therapies. This is particularly important in older patients, who tend to be affected by several co-morbidities, and in whom the cause of reduced exercise tolerance is often more difficult to understand [34]. In view of the differences of age and gender differences in $\mathrm{Vo}_{2 \max }$, numerous equations for age-predicted standards have been published and it is recommended to report peak $\mathrm{VO}_{2}$ also as percentpredicted value [35]. Still, CPET presents some limitations. First, most of the CPET studies on cardiac patients' assessment excluded very old and frail patients: a recent position paper of the European Society of Cardiology underlined the importance of evaluating elderly patients affected by heart failure with CPET using adapted protocols, and recommended attention in the interpretation of the results, given the limited data available [36]. Moreover, CPET is not always possible to realize: if this is the case, Metabolic Equivalents (METs) achieved during Exercise Testing (ET) and distance walked at 6-minute walking testing (6MWT) can be used as a surrogate indicator of exercise performance [37]. The 6MWT is believed to mimic daily functionality, at least in part, and it is generally well-tolerated and safe: since such activity is familiar, it may be more representative of patients' everyday experience [38].
A distance performed $>400 \mathrm{~m}$ is indicative of good functional capacity; in heart failure, a distance $<300$ meters correlates significantly with mortality and re-hospitalization and is a predictor of frailty, while an increase of $50 \mathrm{~m}$ at the repetition of the test is considered clinically significant in all cardiovascular diseases [39]. The ability to perform a $6 \mathrm{MWT}>300 \mathrm{~m}$ is directly related to the mortality in elderly CABG patients before CR [40]. In recent works, the distance traveled at $6 \mathrm{MWT}$ seems to be related to the peak $\mathrm{VO}_{2}$ assessed by CPET but not to survival [41].

The measure of aerobic capacity is essential to prescribe a safe training program, but it is insufficient to evaluate physical efficiency in the elderly. Strength, balance, and flexibility are even more relevant in assessing an older individual's ability to perform common household tasks and remain independent. The Short Physical Performance Battery (SPPB) is an objective tool for measuring the performance of the lower limbs. It demonstrated great sensitivity in identifying and stratifying fragility, and a strong prognostic indicator of the risk of disability, hospitalization, institutionalization, and death [42]. It consists of a series of 3 tests (Gait Speed, Chair Rises, and Tandem Balance); each parameter can get a score from 1 to 4 . A cumulative score $<9 / 12$ is considered indicative of reduced physical performance; a score $<5$ is indicative of frailty. The reduction of Gait Speed is part of Fried's criteria for the definition of "frailty", demonstrating a prognostic value both in the general elderly population and in the elderly cardiac patients; it also demonstrated a strong correlation with the 6MWT, being able to predict the cut-offs of 300 and $400 \mathrm{~m}$ with sensitivity and specificity of $80 \%$ [43]. The main characteristics of physical performance evaluations are summarized in Table 2. Several studies have demonstrated the efficacy of programs in enhancing older patients' functional capacity, variously expressed (peak $\mathrm{VO}_{2}>15 \%$; peak anaerobic threshold $>11 \%$; METs $>3.5-4.3)[35,43,44]$. Moreover, a self-reported measure of physical activity named Physical Activity Scale for the Elderly (PASE), predicts mortality independently of several demographic and clinical variables in elderly patients with advanced heart failure enrolled in CR after heart failure decompensation [45]. However, the majority of these studies enrolled a small proportion of old (75-84 years) and oldestold $(>85)$ patients. In very old patients (mean age $80 \pm 4$ years; range 75-93 years) CR has been demonstrated to increase three indexes of physical performance from baseline to discharge (aerobic power: $\mathrm{VO}_{2}$ peak, $+10.9 \%$; resistance: $6 \mathrm{MWT}+11.0 \%$; muscular strength: peak torque, $+11.5 \%$ ) after acute coronary syndrome or cardiac surgical intervention, particularly in those with poorer baseline performance [16]. In the randomized-control trial CR-AGE, the effect of a comprehensive outpatient-CR program in very old (75$85 \mathrm{yrs}$ ) patients after 2 months of intervention, showed that exercise capacity, as expressed by total work capacity (TWC), was higher in all age patients, while it remained unchanged in the control group. At 6 and 14 months follow up, TWC remained significantly higher than the baseline only in middle-aged CR patients, whereas, with home-based CR, TWC remained higher than baseline in all age groups up to 14 months follow-up [46]. We conducted a prospective, randomized trial (Cardiac Rehabilitation in Advanced aGE: EXercise TRaining and Active follow-up, CR-AGE EXTRA), to assess whether a home-based, 6-month exercise program with monthly follow-up and exercise sessions provides any functional advantage over usual care after a 4 -week outpatient CR program in a large series of patients older than 75 years. The program had a neutral effect on global functional capacity, health-related quality of life, and re-hospitalization rate. Notwithstanding, a relevant finding of our trial was that the CR program in octogenarian patients, that are at increased risk of functional deterioration or with 
a low physical performance after hospitalization, is effective in producing a sustained improvement in functional capacity measures. That is, a well-structured health education program produced both a high adherence rate and a sustained functional benefit over the follow-up [47].

\section{Exercise training protocols}

If we combine the geriatric and the cardiologic evaluation, we can identify different phenotypes of patients and assign them to different paths. For instance, very fit patients, after a post-acute cardiac well-controlled disease, affected by few or absent comorbidities, might be treated in a usual outpatient CR. If a patient was fit before the cardiac event but gets severely disabled on a temporary basis (defined as loss of 3 or more ADL after hospitalization), a tailored program with training sessions of gait and walk, calisthenics exercises and muscular strengthening to recover motor impairment is advisable after the achievement of clinical stability [22]. Exercise training is a key component of a comprehensive CR program and has been traditionally focused on physical activities designed to improve cardiorespiratory fitness. However, older adults CVD can also benefit from physical activities designed to maintain or improve functional independence and tailored around specific needs [48]. Moderate-intensity continuous training (MICT), has traditionally been a foundation of aerobicbased exercise prescriptions resulting in short- and long-term clinical benefits for patients with CVD [49]. High Intensity Interval Training (HIIT) involves repeated bouts of relatively higher intensity exercise, interspersed with periods of lower-intensity recovery. It has recently emerged as an alternative or adjunct strategy, since it has been shown to result in similar or greater improvements in $\mathrm{VO}_{2}$ peak with respect to $\mathrm{MICT}$, even in patients affected by HFrEF. Specifically, it may be superior in improving clinical outcomes for older patients, including quality of life, heart rate response to exercise, and myocardial function. Numerous studies have demonstrated that HIIT is safe even in the elderly. Dun et al proposed short-interval HIIT (10 sets of 1-minute highintensity exercise interspersed with 9 x 2-minute low-intensity intervals) for patients with low exercise capacity or in the initial stage of CR, and medium- ( $8 \times 2$ min interspersed with $7 \times 2$ min) or long- (4 x 4 min interspersed with $3 \times 3$ min) interval HIIT for patients with intermediate or high exercise capacity ( $>5$ METs) [49]. Resistance training (RT) has long been identified as the most promising method for increasing muscle mass and strength among older people. A growing literature has confirmed the effectiveness of resistance training in improving muscle mass, strength, balance, and endurance among the elderly [50]. RT is a form of anaerobic exercise that uses repeated movements against resistance to

Table 1. Frailty scale suggested for clinical use.

\begin{tabular}{lc}
\hline Robust, active, energetic and motivated & Very fit \\
Commonly exercise regularly & Well/managing well \\
$\begin{array}{l}\text { No active disease symptoms, or well controlled medical problems } \\
\text { Occasionally exercise or are very active }\end{array}$ & Vulnerable \\
\hline $\begin{array}{l}\text { Symptoms limit activities, but not dependent for daily help } \\
\text { Feels "slowed up" or tired during the day }\end{array}$ & Mildly/moderately frail \\
$\begin{array}{l}\text { More evident slowing } \\
\text { Needs help in high order IADLa (finances, transportation, heavy housework, medications); progressively impairs shopping and } \\
\text { walking outside alone, meal preparation and housework }\end{array}$ & Severely frail \\
$\begin{array}{l}\text { Completely dependent for personal care from whatever cause (physical or cognitive) } \\
\text { Not at high risk of dying within } 6 \text { months }\end{array}$ & Terminally ill \\
\hline Life expectancy $<6$ months
\end{tabular}

Table 2. Physical performance evaluation.

\begin{tabular}{ll} 
Physical performance evaluation & Main characteristics \\
CardioPulmonary Exercise Test (CPET) & - Gold standard for assessing aerobic capacity \\
& - Identify alterations of the cardiovascular, respiratory or skeletal muscle system, and effects of drug therapies \\
& - Inducible ischemia evaluation and safely exercise prescription \\
& - Strong prognostic valueIt requires specific equipment and experienced staff \\
& - Available assessment of exercise tolerance \\
& - Inducible ischemia evaluation and safely exercise prescription \\
\hline 6-minute walking test (6MWT) & - Available assessment of exercise tolerance \\
& - Representative of patients' everyday experience \\
& - Prognostic value \\
Short Physical Performance Battery (SPPB) & - Performance of the lower limbs \\
& - Quick and easy to assess \\
& - Frailty indicator \\
& - Strong prognostic value
\end{tabular}


stimulate a stronger muscle contraction. It is generally guided by exercise intensity as defined by the single-repetition maximal lift. Gains in strength result from an overload above a minimal threshold of resistance, owing first to neuromuscular adaptation and then by muscular hypertrophy [51]. RT can help to combat frailty by improving several components of the physical function, including muscular strength, walking endurance, and balance. It brings significant improvements in the activity of daily living even without a change in aerobic capacity, which may be particularly relevant in perceived quality of life. It has shown to safely improve performance in older women, both with coronary heart disease and with systolic HF [51]. Supervised RT seems to be quite safe, even among the elderly in CR. Combined RT and aerobic exercise have proven to be more effective in improving peak $\mathrm{VO}_{2}$, muscle strength, endurance, and quality of life if compared with RT or aerobic exercised alone [51].

\section{Conclusions}

With the progressive aging of the cardiac population, the CR programs must face the challenge to manage more complex patients and to assess the presence of geriatric syndromes. There is a consensus that interventions should be individual-tailored and aimed at both preventing disability and preserving residual functional capacity. Very old and frail patients are often excluded, even though they seem to get an even greater potential benefit of CR than the "healthier" old patients. Since studies about CR programs in very old and frail patients are still few, a great effort is necessary to fill this gap. Although it remains woefully underutilized, CR appears to have favorable effects on various aspects of health among the elderly population. The reduced participation of elderly patients in formal CR programs can be attributed to multiple reasons. Improved efforts are needed to encourage eligible patients to attend and complete these programs. As the population ages, an increasing proportion of inactive, low-fit adults aged 85 years and older, will be CR candidates, a cohort that appears to be the primary beneficiary of an exercise intervention. Specific processes of care (both concerning exercise and broader CR goals) can likely be refined to meet the needs of the aging population of vulnerable elders.

\section{References}

1. Anderson L, Thompson DR, Oldridge N, et al. Exercise-based cardiac rehabilitation for coronary heart disease. Cochrane Database Syst Rev 2016;CD001800

2. Cesari M, Calvani R, Marzetti E. Frailty in older persons. Clin Geriatr Med 2017;33:293-303.

3. Hoogendijk EO, Afilalo J, Ensrud KE, et al. Frailty: implications for clinical practice and public health. Lancet 2019; 394:1365-75.

4. Romero-Ortuno R, Kenny RA. The frailty index in Europeans: association with age and mortality. Age Ageing 2012;41:684-9.

5. Dent E, Martin FC, Bergman H, et al. Management of frailty: opportunities, challenges, and future directions. Lancet 2019;394:1376-86.

6. Fried LP, Tangen CM, Walston J, et al. Frailty in older adults: evidence for a phenotype. J Gerontol A Biol Sci Med Sci 2001;56:M146-56.
7. Rockwood K, Song X, MacKnight C, et al. A global clinical measure of fitness and frailty in elderly people. CMAJ 2005; 173:489-95.

8. Vigorito C, Abreu A, Ambrosetti M, et al. Frailty and cardiac rehabilitation: A call to action from the EAPC Cardiac Rehabilitation Section. Eur J Prev Cardiol 2017;24:577-90.

9. Walston J, Buta B, Xue QL. Frailty screening and interventions: Considerations for clinical practice. Clin Geriatr Med 2018;34:25-38.

10. Afilalo J. Evaluating and treating frailty in cardiac rehabilitation. Clin Geriatr Med 2019;35:445-57.

11. Pedretti RFE, Fattirolli F, Griffo R, et al. Cardiac Prevention and Rehabilitation "3.0": From acute to chronic phase. Position Paper of the 1talian Association for Cardiovascular Prevention and Rehabilitation (GICR-IACPR). Monaldi Arch Chest Dis 2018;88:1004.

12. Goyal P, Gorodeski EZ, Marcum ZA, Forman DE. Cardiac rehabilitation to optimize medication regimens in heart failure. Clin Geriatr Med 2019;35:549-60.

13. Guigoz Y, Vellas B, Garry PJ. Assessing the nutritional status of the elderly: The Mini Nutritional Assessment as part of geriatric evaluation. Nutr Rev 1996;54:S59-65.

14. Baker JP, Detsky AS, Wesson DE, et al. Nutritional assessment: a comparison of clinical judgement and objective measurements. N Engl J Med 1982;306:969-72.

15. Sullivan DH, Patch GA, Walls RC, Lipschitz. Impact of nutritional status on morbility and mortality in a select population of geriatric rehabilitation patients. Am J Clin Nutr 1990;51:749-58.

16. Baldasseroni S, Pratesi A, Francini S, et al. Cardiac rehabilitation in very old adults: Effect of baseline functional capacity on treatment effectiveness. J Am Geriatr Soc 2016;64:1640-5.

17. Giallauria F, Vigorito C, Tramarin R, et al. Cardiac rehabilitation in very old patients: data from the Italian Survey on Cardiac Rehabilitation-2008 (ISYDE-2008) -- official report of the Italian Association for Cardiovascular Prevention, Rehabilitation, and Epidemiology. J Gerontol A Biol Sci Med Sci 2010;65:1353-61.

18. Ziegelstein RC, Fauerbach JA, Stevens SS, et al. Patients with depression are less likely to follow recommendations to reduce cardiac risk during recovery from a myocardial infarction. Arch Intern Med 2000;160:1818-23.

19. Frasure-Smith N, Lesperance F, Talajic M. Depression and 18month prognosis after myocardial infarction. Circulation 1995;91:999-1005.

20. Marchionni N, Fattirolli F, Fumagalli S, et al. Determinants of exercise tolerance after acute myocardial infarction in older persons. J Am Geriatr Soc 2000;48:146-53.

21. Richards SH, Anderson L, Jenkinson CE, et al. Psychological interventions for coronary heart disease. Cochrane Database Syst Rev 2017;CD002902.

22. Schopfer DW, Forman DE. Cardiac rehabilitation in older adults. Can J Cardiol 2016;32:1088-96.

23. Suaya JA, Stason WB, Ades PA, et al. Cardiac rehabilitation and survival in older coronary patients. J Am Coll Cardiol 2009;54:25-33.

24. Davies EJ, Moxham T, Rees K, et al. Exercise based rehabilitation for heart failure. Cochrane Database Syst Rev 2010:CD003331.

25. Taylor RS, Sagar VA, Davies EJ, et al. Exercise-based rehabilitation for heart failure. Cochrane Database Syst Rev 2014;CD003331. 
26. Eichler S, Salzwedel A, Reibis R, et al. Multicomponent cardiac rehabilitation in patients after transcatheter aortic valve implantation: Predictors of functional and psychocognitive recovery. Eur J Prev Cardiol 2017;24:257-64.

27. Cesari M, Onder G, Zamboni V, et al. Physical function and self-rated health status as predictors of mortality: results from longitudinal analysis in the ilSIRENTE study. BMC Geriatr 2008;8:34.

28. Keeney T, Fox AB, Jette DU, Jette A. Functional trajectories of persons with cardiovascular disease in late life. J Am Geriatr Soc 2019;67:37-42.

29. Plichart M, Barberger-Gateau P, Tzourio C, et al. Disability and incident coronary heart disease in older community-dwelling adults: The Three-City Study. J Am Geriatr Soc 2010;58:636-42.

30. Levine DA, Davydow DS, Hough CL, et al. Functional disability and cognitive impairment after hospitalization for myocardial infarction and stroke. Circ Cardiovasc Qual Outcomes 2014;7:863-71.

31. Forman DE, Arena R, Boxer R, et al. Prioritizing functional capacity as a principal end point for therapies oriented to older adults with cardiovascular disease: A scientific statement for healthcare professionals from the American Heart Association. Circulation 2017;135:e894-918.

32. Cacciatore F, Abete P. Cardiac rehabilitation in the elderly patients. In: Masiero S. Rehabilitation for elderly patients. Springer; 2017.

33. Moorhouse P, Rockwood K. Frailty and its quantitative clinical evaluation. J R Coll Physicians Edinb 2012;42:333-40.

34. Malhotra R, Bakken K, D'Elia E, Lewis GD. Cardiopulmonary exercise testing in heart failure. JACC Heart Fail 2016;4:607-16.

35. Arena R, Myers J, Williams MA, et al. Assessment of functional capacity in clinical and research settings: a scientific statement from the American Heart Association Committee on Exercise, Rehabilitation, and Prevention of the Council on Clinical Cardiology and the Council on Cardiovascular Nursing. Circulation 2007;116:329-43.

36. Corrà U, Agostoni PG, Anker SD, et al. Role of cardiopulmonary exercise testing in clinical stratification in heart failure. A position paper from the Committee on Exercise Physiology and Training of the Heart Failure Association of the European Society of Cardiology. Eur J Heart Fail 2018;20:3-15.

37. Forman DE, Fleg JL, Kitzman DW, et al. 6-min walk test provides prognostic utility comparable to cardiopulmonary exercise testing in ambulatory outpatients with systolic heart failure. J Am Coll Cardiol 2012;60:2653-61.

38. Olsson LG, Swedberg K, Clark AL, et al. Six minute corridor walk test as an outcome measure for the assessment of treatment in randomized, blinded intervention trials of chronic heart failure: a systematic review. Eur Heart J 2005;26:778-93.

39. Boxer R, Kleppinger A, Ahmad A, et al. The 6-minute walk is associated with frailty and predicts mortality in older adults with heart failure. Congest Heart Fail 2310;16:208-13.

40. Cacciatore F, Abete P, Mazzella F, et al. Six-minute walking test but not ejection fraction predicts mortality in elderly patients undergoing cardiac rehabilitation following coronary artery bypass grafting. Eur J Prev Cardiol 2012;19:1401-9.

41. Kamiya K, Hamazaki N, Matsue Y, et al. Gait speed has comparable prognostic capability to six-minute walk distance in older patients with cardiovascular disease. Eur J Prev Cardiol 2018;25:212-9.

42. Chiarantini D, Volpato S, Sioulis F, et al. Lower extremity performance measures predict long-term prognosis in older patients hospitalized for heart failure. J Card Fail 2010;16:390-5.

43. Lavie CJ, Milani RV. Benefits of cardiac rehabilitation and exercise training programs in elderly coronary patients. Am J Geriatr Cardiol 2001;10:323-7.

44. Lavie CJ, Milani RV. Effects of cardiac rehabilitation programs on exercise capacity, coronary risk factors, behavioral characteristics, and quality of life in a large elderly cohort. Am J Cardiol 1995; 76:177-9.

45. Cacciatore F, Amarelli C, Ferrara N, et al. Protective effect of physical activity on mortality in older adults with advanced chronic heart failure: A prospective observational study. Eur J Prev Cardiol 2019;26:481-8.

46. Marchionni N, Fattirolli F, Fumagalli S, et al. Improved exercise tolerance and quality of life with cardiac rehabilitation of older patients after myocardial infarction: results of a randomized, controlled trial. Circulation 2003;107:2201-6.

47. Pratesi A, Baldasseroni S, Burgisser C, et al. Long-term functional outcomes after cardiac rehabilitation in older patients. Data from the Cardiac Rehabilitation in Advanced aGE: EXercise TRaining and Active follow-up (CR-AGE EXTRA) randomised study. Eur J Prev Cardiol 2019;26:1470-8.

48. Fiatarone Singh MA. Tailoring assessments and prescription in cardiac rehabilitation for older adults: The relevance of geriatric domains. Clin Geriatr Med 2019;35:423-43.

49. Dun Y, Thomas RJ, Medina-Inojosa JR, et al. High-intensity interval training in cardiac rehabilitation: Impact on fat mass in patients with myocardial infarction. Mayo Clin Proc 2019;94: 1718-30.

50. Giallauria F, Cittadini A, Smart NA, Vigorito C. Resistance training and sarcopenia. Monaldi Arch Chest Dis 2016;84:738.

51. Khadanga S, Savage PD, Ades PA. Resistance training for older adults in cardiac rehabilitation. Clin Geriatr Med 2019;35: 459-68. 